\title{
Noninvasive ventilation in stable chronic obstructive pulmonary disease
}

\section{To the Editor:}

Chronic obstructive pulmonary disease (COPD) with severe emphysema is a continuously progressing disease, resulting in respiratory failure. Pharmacological treatment may be optimised in the last years, but only the introduction of long-term oxygen treatment has revealed an improvement in survival for patients with advanced COPD [1].

Noninvasive positive pressure ventilation (NPPV) is a new approach to directly correct ventilatory insufficiency. The beneficial effects of NPPV during acute exacerbation of COPD are well documented [2], and NPPV is frequently applied in these patients today [3]. However, domiciliary longterm use of NPPV by patients with severe COPD remains controversial.

In 1996, the Italian Rehabilitation and Chronic Care Study Group started a prospective, randomised, controlled, multicentre trial to evaluate NPPV in severe chronic stable COPD. The main outcome parameters were arterial blood gases, hospital and intensive care unit admissions and length of stay, health related quality of life, and exercise tolerance. The results of this study had been recently presented in the European Respiratory Journal [4]. The conclusions drawn are not in favour of long-term use of NPPV in severe COPD patients. Apart from a quality of life assessment (Maugeri Foundation Respiratory Questionnaire (MRF-28)), none of the other outcome parameters could be significantly improved. The reason for this should be sought in the "ventilator setting" instructions of the protocol: ventilator settings were titrated individually for each patient with the target of maximally tolerated inspiratory pressures and expiratory pressures in the range of $2-5 \mathrm{~cm} \cdot \mathrm{H}_{2} \mathrm{O}$. However, a reduction of baseline carbon dioxide arterial tension by $\geqslant 5 \%$ during $\geqslant 1 \mathrm{~h}$ of daytime NPPV had been considered an effective treatment (E. Clini, Fondazione Villa Pineata ONLUS, Italy, personal communication). This resulted in a mean inspiratory pressure of $14 \pm 3 \mathrm{~cm} \cdot \mathrm{H}_{2} \mathrm{O}$ and a mean expiratory pressure of $2 \pm 1 \mathrm{~cm} \cdot \mathrm{H}_{2} \mathrm{O}$.

The findings of the study by CLINI et al. [4] are in accordance with the survival and hospital admission rates reported by CASANOVA et al. [5], who applied NPPV in a similar cohort of COPD patients with a mean inspiratory pressure of $12 \mathrm{~cm} \cdot \mathrm{H}_{2} \mathrm{O}$ and a mean expiratory pressure of $4 \mathrm{~cm} \cdot \mathrm{H}_{2} \mathrm{O}$. However, in $1995 \mathrm{MeECHAM-JONES} \mathrm{et} \mathrm{al.} \mathrm{[6]} \mathrm{demon-}$ strated significant improvements of blood gases, sleep quality and quality of life in hypercapnic COPD patients after 3 months of NPPV with a mean inspiratory pressure of $20 \mathrm{~cm} \mathrm{H} \mathrm{H}_{2} \mathrm{O}$.

It is reasonable to speculate that the applied pressures in the study by MEECHAM-JONES et al. [6] resulted in better gas exchange and unloading of the ventilatory muscles. NPPV might otherwise be "underdosed" in patients with severe COPD and the therapeutic goal of correcting the underlying pathophysiology is missed. It can be troublesome to establish NPPV in patients with severe COPD and with growing ventilatory pressures it becomes more and more difficult to keep the patient compliant. The balance between therapeutic requirements and patients' comfort is a challenge for both patient and staff.

Further studies confirming and extending the findings of MeEcham-Jones et al. [6] findings to define the role of noninvasive positive pressure ventilation as a treatment option for patients with severe chronic obstructive pulmonary disease are needed.

\author{
T. Köhnlein, T. Welte \\ Otto-von-Guericke-Universität Magdeburg, Pulmonary and \\ Intensive Care Medicine, Leipziger Strasse 44, D-39120 Mage- \\ burg, Germany.
}

\section{References}

1. Report of the Medical Research Council Working Party. Long-term domiciliary oxygen in chronic corpulmonale complicating chronic bronchitis and emphysema. Lancet 1981; 1: 681-685.

2. Brochard L, Mancebo J, Wysocki M, et al. Noninvasive ventilation for acute exacerbations of chronic obstructive pulmonary disease. $N$ Engl J Med 1995; 333: 817-822.

3. Keenan SP, Kernerman PD, Cook DJ, Martin CM, McCornmack D, Sibbald WJ. Effect of noninvasive positive pressure ventilation on mortality in patients admitted with acute respiratory failure: A meta-analysis. Crit Care Med 1997; 25: 1685-1692.

4. Clini E, Sturani C, Rossi A, et al. The Italian mulitcentre study on noninvasive ventilation in chronic obstructive pulmonary disease patients. Eur Respir J 2002; 20: 529-538.

5. Casanova C, Celli BR, Tost L, et al. Long-term controlled trial of nocturnal nasal positive pressure ventilation in patientes with severe COPD. Chest 2000; 118: 1582-1590.

6. Meecham Jones DJ, Paul EA, Jones PW, Wedzicha JA. Nasal pressure support ventilation plus oxygen compared with oxygen therapy alone in hypercapnic COPD. Am J Respir Crit Care Med 1995; 152: 538-544.

\section{From the authors:}

We would like to thank T. Koehnlein and T. Welte for their comments on our paper dealing with the use of noninvasive positive pressure ventilation (NPPV) in chronic obstructive pulmonary disease (COPD) patients as recently published in the European Respiratory Journal [1].

They essentially stated that the results of that multicentre study were negative, by observing that the lack of response in most of the physiological and clinical variables could be ascribed to the "relatively" low level of assistance delivered, as a mean, by the ventilator.

However, we keep on thinking about a possible positive role for NPPV in a clearly selected sample of severe COPD patients. Indeed, the long-term protective effect on blood gases (namely, arterial carbon dioxide tension $\left(\mathrm{Pa}, \mathrm{CO}_{2}\right)$ ) while breathing oxygen (which is the most common daily condition for those patients) observed in the NPPV group has indirectly shown the actual physiological value of this study [1]. Although the pathophysiological reason for this behaviour (different from that observed in the control patients) cannot be drawn from this clinical study, it is reasonable to hypothesise that a probable long-term protection against hypoventilation, as demonstrated previously [2], also still occurred at a lower level of assistance.

Nevertheless, we completely agree with T. Koehnlein and $\mathrm{T}$. Welte in that increasing pressure levels might decrease compliance and affect the physiological response (mainly related to the increasing leaks) of the ventilatory treatment. 